\title{
HAMILTONIAN FORMULATION OF QED IN THE SUPERAXIAL GAUGE
}

\author{
Horacio O. GIROTTI ${ }^{1}$ \\ Instituto de Fisica, Universidade Federal do Rio Grande do Sul, 90000 Porto Alegre, RS, Brasil \\ and
}

Heinz J. ROTHE
Institut für Theoretische Physik, Universität Heidelberg, D6900 Heidelberg, Germany

Received 19 April 1982

We present a hamiltonian formulation of QED in a fully fixed axial gauge. The equal-time commutators for all field variables are computed and are shown to lead to the correct equations of motion. The constraints and gauge conditions hold as strong operator relations.

It has long been known [1] that gauge theories fall into the class of constrained systems and that only the hamiltonian formalism provides an entirely reliable scheme for quantizing such theories [1-3].

The implementation of a quantum hamiltonian dynamics for abelian as well as non-abelian gauge field theories has been carried out in the case of the Coulomb gauge [4] ${ }^{\neq 1}$. On the other hand, there have been several attempts to obtain a hamiltonian formulation in the axial gauge $A^{3}=0[6,7]$. This last mentioned gauge has proved to be very useful in discussing non-abelian gauge theories. It is the purpose of this paper to present the hamiltonian formulation of QED in the fully fixed axial gauge specified by

$A^{3}\left(x^{0}, x^{1}, x^{2}, x^{3}\right)=0, \quad A^{1}\left(x^{0}, x^{1}, x^{2}, x_{(0)}^{3}\right)=A^{2}\left(x^{0}, x_{(0)}^{1}, x^{2}, x_{(0)}^{3}\right)=0$,

$A^{0}\left(x^{0}, x_{(0)}^{1}, x_{(0)}^{2}, x_{(0)}^{3}\right)=-\left.\left(\partial^{j} / \nabla^{2}\right) \pi_{j}\left(x^{0}, x\right)\right|_{x=x_{(0)}}$.

Here, the $A^{\mu}$ 's $(\mu=0,1,2,3)$ are the gauge potentials while the $\pi_{j}$ 's $(i=1,2,3)$ are the momenta canonically conjugate to the $A^{i}$ s. Furthermore $x_{(0)}=\left(x_{(0)}^{1}, x_{(0)}^{2}, x_{(0)}^{3}\right)$ denotes some arbitrary fixed point. Summation convention on repeated indices must be understood. Latin indices sum from 1 to 3 unless otherwise is specified. Our space-time metric is $g_{00}=-g_{11}=-g_{22}=-g_{33}=+1, g_{\mu \nu}=0$ if $\mu \neq \nu$.

One can easily convince oneself that the conditions (1) fix the gauge uniquely, i.e., up to a global gauge transformation. Within the set of gauges $A^{3}=0$ we have chosen a very particular one to which we shall refer to as the superaxial gauge. If instead of eq. (1c) had we chosen $A^{0}\left(x^{0}, x_{(0)}^{1}, x_{(0)}^{2}, x_{(0)}^{3}\right)=0$ then our gauge would coincide with that employed by Halpern in a different context [8]. As we shall see condition (1c) plays an important role in the process of obtaining a consistent hamiltonian formulation of QED.

Our strategy consists in observing that the gauge (1) can be reached from the Coulomb gauge via the following operator gauge transformation

$A^{\mu}(x)=A^{\mu, \mathrm{C}}(x)+\partial^{\mu} \Lambda(x), \quad \psi(x)=\exp [\mathrm{i} e \Lambda(x)] \psi^{\mathrm{C}}(x)$,

\footnotetext{
1 Supported in part by Conselho Nacional de Desenvolvimento Científico e Tecnológico (CNPq), Brasil.

$\neq 1$ See also ref. [5] for a formulation of the problem in the Dirac bracket formalism.
} 
where

$\Lambda(x)=\int_{x_{(0)}^{3}}^{x^{3}} \mathrm{~d} x^{\prime 3} A^{3, \mathrm{C}}\left(x^{0}, x^{1}, x^{2}, x^{\prime 3}\right)+\int_{x_{(0)}^{1}}^{x^{1}} \mathrm{~d} x^{11} A^{1, \mathrm{C}_{(}}\left(x^{0}, x^{\prime 1}, x^{2}, x_{(0)}^{3}\right)+\int_{x_{(0)}^{2}}^{x^{2}} \mathrm{~d} x^{\prime 2} A^{2, \mathrm{C}}\left(x^{0}, x_{(0)}^{1}, x^{\prime 2}, x_{(0)}^{3}\right)$,

and $e$ is the electron charge. We have denoted all field variables belonging to the Coulomb gauge by the superscript "C", while field variables without gauge identification are understood to belong to the superaxial gauge. The nonvanishing equal-time commutators (ETC's) in the Coulomb gauge are known to read [4,5]

$\left[A^{i, \mathrm{C}}\left(x^{0}, \boldsymbol{x}\right), \pi_{j}\left(x^{0}, \boldsymbol{y}\right)\right]=\mathrm{i}\left(\delta^{i j}-\partial_{x}^{i} \partial_{x}^{j} / \nabla_{x}^{2}\right) \delta(\boldsymbol{x}-\boldsymbol{y}), \quad\left\{\psi^{\mathrm{C}}\left(x^{0}, \boldsymbol{x}\right), \pi_{\psi}^{\mathrm{C}}\left(x^{0}, \boldsymbol{y}\right)\right\}=\mathrm{i} \delta(\boldsymbol{x}-\boldsymbol{y})$,

$\left[\pi_{j}\left(x^{0}, x\right), \psi^{\mathrm{C}}\left(x^{0}, y\right)\right]=-e \psi^{\mathrm{C}}\left(x^{0}, y\right)\left(\partial_{y}^{j} / \nabla_{y}^{2}\right) \delta(y-x), \quad\left[\pi_{j}\left(x^{0}, x\right), \pi_{\psi}^{\mathrm{C}}\left(x^{0}, y\right)\right]=e \pi_{\psi}^{\mathrm{C}}\left(x^{0}, y\right)\left(\partial_{y}^{j} / \nabla_{y}^{2}\right) \delta(y-x)$,

where $\pi_{\psi}$ is the canonical momentum conjugate to the spinor field $\psi$. No gauge superscript has been attached to the $\pi_{j}$ 's since they are gauge invariant quantities. Therefore, it follows from eqs. (2) - (5) that the ETC's in the superaxial gauge can be found from the corresponding ones in the Coulomb gauge. In this respect notice that

$\left[A^{i, \mathrm{C}}\left(x^{0}, x\right), \psi^{\mathrm{C}}\left(x^{0}, y\right)\right]=0$,

guarantees that there are no ordering problems in eq. (3). Moreover, we emphasize that our particular choice of condition (1c) secures that $\Lambda$ is a local function of time. Indeed, any other choice for $A^{0}\left(x^{0}, x_{(0)}\right)$ would lead to a $\Lambda$ which is non-local in time. In particular, if $(1 \mathrm{c})$ is replaced by

$A^{0}\left(x^{0}, x_{(0)}^{1}, x_{(0)}^{2}, x_{(0)}^{3}\right)=0$,

then, the gauge operator function $\Lambda$ connecting the Coulomb gauge with the superaxial gauge differs from that given in eq. (4) by the term

$-\int_{x_{(0)}^{0}}^{x^{0}} \mathrm{~d} x^{\prime 0} A^{0, \mathrm{C}}\left(x^{\prime 0}, x_{(0)}\right)$

where

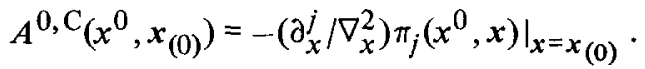

This is the reason for having chosen condition (1c).

For the non-vanishing ETC's in the superaxial gauge we obtain

$$
\begin{aligned}
& {\left[A^{1}\left(x^{0}, x\right), \pi_{j}\left(x^{0}, y\right)\right]=\mathrm{i} \delta^{1 j} \delta\left(x^{1}-y^{1}\right) \delta\left(x^{2}-y^{2}\right)\left[\delta\left(x^{3}-y^{3}\right)-\delta\left(x_{(0)}^{3}-y^{3}\right)\right]} \\
& \quad+\mathrm{i} \delta^{3 j}\left[\partial_{x}^{1} \delta\left(x^{1}-y^{1}\right)\right] \delta\left(x^{2}-y^{2}\right) \Delta\left(x^{3}, x_{(0)}^{3} ; y^{3}\right), \\
& {\left[A^{2}\left(x^{0}, x\right), \pi_{j}\left(x^{0}, y\right)\right]=\mathrm{i} \delta^{1 j}\left[\partial_{x}^{2} \delta\left(x^{2}-y^{2}\right)\right] \delta\left(x_{(0)}^{3}-y^{3}\right) \Delta\left(x^{1}, x_{(0)}^{1} ; y^{1}\right)} \\
& \quad+\mathrm{i} \delta^{2 j} \delta\left(x^{2}-y^{2}\right)\left[\delta\left(x^{1}-y^{1}\right) \delta\left(x^{3}-y^{3}\right)-\delta\left(x_{(0)}^{1}-y^{1}\right) \delta\left(x_{(0)}^{3}-y^{3}\right)\right] \\
& \quad+\mathrm{i} \delta^{3 j} \delta\left(x^{1}-y^{1}\right)\left[\partial_{x}^{2} \delta\left(x^{2}-y^{2}\right)\right] \Delta\left(x^{3}, x_{(0)}^{3} ; y^{3}\right), \\
& \left\{\psi\left(x^{0}, x\right), \pi_{\psi}\left(x^{0}, y\right)\right\}=\mathrm{i} \delta(x-y), \\
& {\left[\pi_{j}\left(x^{0}, y\right), \psi\left(x^{0}, x\right)\right]=-e \psi\left(x^{0}, x\right)\left(\partial_{x}^{j} / \nabla_{x}^{2}\right) \delta(x-y)+e \Omega_{j}\left(x, y, x_{(0)}\right) \psi\left(x^{0}, x\right),} \\
& {\left[\pi_{j}\left(x^{0}, y\right), \pi_{\psi}\left(x^{0}, x\right)\right]=e \pi_{\psi}\left(x^{0}, x\right)\left(\partial_{x}^{j} / \nabla_{x}^{2}\right) \delta(x-y)-e \Omega_{j}\left(x, y, x_{(0)}\right) \pi_{\psi}\left(x^{0}, x\right),}
\end{aligned}
$$

where 
$\Delta\left(x^{i}, x_{(0)}^{i} ; y^{i}\right)=\int_{x_{(0)}^{i}}^{x^{i}} \mathrm{~d} x^{\prime i} \delta\left(x^{\prime i}-y^{i}\right)$,

and

$$
\begin{aligned}
& \Omega_{j}\left(\boldsymbol{x}, \boldsymbol{y}, \boldsymbol{x}_{(0)}\right)=\left(\delta^{j 1}-\partial_{y}^{j} \partial_{y}^{1} / \nabla_{y}^{2}\right) \delta\left(y^{2}-x^{2}\right) \delta\left(x_{(0)}^{3}-y^{3}\right) \Delta\left(x^{1}, x_{(0)}^{1} ; y^{1}\right) \\
& \quad+\left(\delta^{j 2}-\partial_{y}^{j} \partial_{y}^{2} / \nabla_{y}^{2}\right) \delta\left(x_{(0)}^{1}-y^{1}\right) \delta\left(x_{(0)}^{3}-y^{3}\right) \Delta\left(x^{2}, x_{(0)}^{2} ; y^{2}\right) \\
& \quad+\left(\delta^{j 3}-\partial_{y}^{j} \partial_{y}^{3} / \nabla_{y}^{2}\right) \delta\left(x^{1}-y^{1}\right) \delta\left(x^{2}-y^{2}\right) \Delta\left(x^{3}, x_{(0)}^{3} ; y^{3}\right) .
\end{aligned}
$$

The ETC's involving $A^{0}$ can be similarly computed but since they play no role in the hamiltonian dynamics they will not be given here. It can readily be checked that our ETC's [eq. (7)] are compatible with the Gauss law, i.e.,

$\partial^{j} \pi_{j}-\mathrm{i} e \pi_{\psi} \cdot \psi=0$,

and with the gauge conditions (1) as strong operator relations. The "dot" in eq. (10) symbolizes

$\pi_{\psi}(x) \cdot \psi(x)=\frac{1}{2}\left[\pi_{\psi}(x), \psi(x)\right]$.

In order to find the quantum equations of motion we must now specify the hamiltonian operator $(H)$ in terms of axial gauge field variables. The hamiltonian operator of QED generating the correct equations of motion for the Coulomb field variables is known [4-9]. Furthermore, it is invariant under the operator gauge transformation (2)-(4) and hence is given by

$H=\int \mathrm{d}^{3} z\left[\frac{1}{2} \pi_{j} \pi_{j}+\frac{1}{4} F^{i j} F^{i j}+\frac{1}{2} \pi_{\psi} \cdot \gamma^{0} \gamma^{k}\left(\partial^{k} \psi\right)-\frac{1}{2}\left(\partial^{k} \pi_{\psi}\right) \cdot \gamma^{0} \gamma^{k} \psi-\mathrm{i} m \pi_{\psi} \cdot \gamma^{0} \psi-\mathrm{i} e \pi_{\psi} \cdot \gamma^{0} \gamma^{k} \psi A^{k}\right]$.

After a rather tedious calculation one arrives at the following equations of motion

$\dot{A}^{j}=\pi_{j}+\partial^{j} A^{0}, \quad j=1,2 ; \quad \dot{A}^{3}=0 ; \quad \dot{\pi}_{j}=\partial^{k} F^{k j}+i e \pi_{\psi} \cdot \gamma^{0} \gamma^{j} \psi, \quad j=1,2,3 ;$

$\dot{\psi}=-\mathrm{i} e \gamma^{0} \gamma^{k} A^{k} \psi-\mathrm{i} m \gamma^{0} \psi+\mathrm{i} e A^{0} \psi+\gamma^{0} \gamma^{k} \partial^{k} \psi ; \quad \dot{\pi}_{\psi}=\mathrm{i} e \pi_{\psi} \gamma^{0} \gamma^{k} A^{k}+\mathrm{i} m \pi_{\psi} \gamma^{0}-\mathrm{i} e \pi_{\psi} A^{0}+\left(\partial^{k} \pi_{\psi}\right) \gamma^{0} \gamma^{k}$,

where

$$
\begin{aligned}
& A^{0}\left(x^{0}, x^{1}, x^{2}, x^{3}\right)=\int_{x_{(0)}^{3}}^{x^{3}} \mathrm{~d} x^{\prime 3} \pi_{3}\left(x^{0}, x^{1}, x^{2}, x^{\prime 3}\right)+\int_{x_{(0)}^{1}}^{x^{1}} \mathrm{~d} x^{\prime 1} \pi_{1}\left(x^{0}, x^{\prime 1}, x^{2}, x_{(0)}^{3}\right) \\
& \quad+\int_{x_{(0)}^{2}}^{x^{2}} \mathrm{~d} x^{\prime 2} \pi_{2}\left(x^{0}, x_{(0)}^{1}, x^{\prime 2}, x_{(0)}^{3}\right)-\left.\left(\partial_{x}^{j} / \nabla_{x}^{2}\right) \pi_{j}\left(x^{0}, x\right)\right|_{x=x_{(0)}},
\end{aligned}
$$

which is precisely the expression following after some trivial manipulations from eqs. (2) and (4). From the equations of motion (13) one sees immediately that the Gauss law (10) and the gauge conditions (1) are persistent in time.

Finally we wish to comment about the structure of the ETC's presented in this paper and to compare our results with some previous attempts for obtaining a hamiltonian formulation of a gauge theory in the axial gauge. One verifies that the ETC's given in eq. (7) are all consistent with the electric and magnetic field vanishing at spatial infinity and hence our hamiltonian (12) is a well defined object. Thus, Schwinger's criticism [10] of the original formulation of the axial gauge by Arnowitt and Fickler [6] does not apply to our case. We also remark that in the formulation of Chodos [7] the ETC's involving the electric field have support at spatial infinity and therefore they 
are not compatible with vanishing electric and magnetic field at spatial infinity.

In conclusion, we have shown that the quantum hamiltonian dynamics of QED in the superaxial gauge can be implemented in a satisfactory way, with all constraints (Gauss law) and gauge conditions appearing as strong operator relations.

One of us (H.J.R.) would like to thank the DAAD and the CNPq and Professor B. Liberman for the kind hospitality extended to him during his stay at the Instituto de Fisica, UFRGS, where part of this work has been done.

\section{References}

[1] P.A.M. Dirac, Lectures on quantum mechanics, Belfer Graduate School of Science, Yeshiva University (New York, NY, 1964).

[2] L.D. Faddeev, Theor. Mat. Fiz. 1 (1963) 3 [English translation Theor. Mat. Phys. 1 (1970) 1].

[3] E.S. Fradkin and G.A. Vilkovisky, preprint CERN TH.2332 (1977).

[4] J. Schwinger, Phys. Rev. 125 (1962) 1043; 127 (1962) 324;130 (1963) 406.

[5] H.O. Girotti and K.D. Rothe, preprint IFUFRGS (1982).

[6] R.L. Arnowitt and S.I. Fickler, Phys. Rev. 127 (1962) 1821.

[7] A. Chodos, Phys. Rev. D17 (1978) 2624.

[8] M.B. Halpern, Phys. Rev. D19 (1979) 517.

[9] H.O. Girotti and K.D. Rothe, Lett. Nuovo Cimento 31 (1981) 545.

[10] J. Schwinger, Phys. Rev. 130 (1963) 402. 